\title{
Improve the Performance of the Work of the Restaurant Using PC Touch Screen
}

\author{
Nibras Othman Abdul Wahid*
}

Department of Mathematics and Computer Science, Graduate School of Natural and Applied Sciences, Ministry of Higher Education and Scientific Research, Iraq

\begin{abstract}
The growth of wireless technology and tablet PC in this era is creating a great impact on our lives. Some early efforts have been made to combine and utilize both of these technologies in advancement of hospitality industry. Nowadays, the majority of restaurants are still operating in an old fashioned way, by using pen and paper to register the orders of customers. The problem using "traditional menu" is probability of paper lost high and misinterprets the handwriting of order. Ours is designed to overcome this problem. By using "tablet PC", the customer can send orders to the cooking room and cashier in a fast and easy way. TSIR can also give customer feedback to restaurant staff. The methodology that has been used in this paper is based on wireless communication (Wi-Fi). This system is developed by using (visual basic 6.0 and SQL server 2000). This system makes the food ordering process easier. This system, implements wireless data access to SQL server. The Windows 7 application on customer tablet pc will have all the menu details. The restaurant manager can manage the menu modifications easily, via adding and removing items.
\end{abstract}

Keywords: Restaurant automation, Wireless food ordering system, Tablet PC, Windows application, Wi-Fi

\section{Introduction}

Some problems, in most restaurants are still used paper and pen to request the orders to customers. Must customer wait, until the waiter comes to register the order from customer. This process takes time from customer. Sometimes, the waiter forgets or he recording the order is mistake on the paper. Another problem traditional menu in restaurants, it not has more details about food for example Calories, food image and time of prepare. We aimed to solve this problem by designing a user interface for a new ordering system. With the new system, the customers would be able to order food from their table using a touch screen (TS) without waiter [1]. When customer order food and press done button in (TS) this bill do to cashier, this bill have more information about food items, price, table number and date/ time. Use this system to make order food easier and faster by use touch screen.

This paper presents use tablet pc wireless communication technologies to realize a wireless food ordering system. In this system, it implements wireless data access to the server and food ordering functions through tablet PC [2].

A wireless LAN (WLAN, Wireless Local Area Network) is a flexible data communication system implemented as an extension to or as an alternative for, a wired LAN within a building or campus [2]. The Institute of Electronics Engineers (IEEE) is one of the most influential standards bodies in the world for information technology. IEEE has created many widely adopted standards, such as port-based Network Access Control (IEEE 802.1X), Ethernet (IEEE802.3), Fire wire (IEEE1394), and wireless LAN (IEEE802.11). The 802.11 standards and its variant wireless LAN standards are now the foundation used by virtually all wireless LAN vendors and production in the world [3]. The IEEE 802.11 group of standards specifies the technologies for wireless LANs. 802.11 standards use the Ethernet protocol and CSMA/CA (carrier sense multiple access with collision avoidance) for path sharing and include an encryption method, the Wired Equivalent Privacy algorithm. The $802.11 \mathrm{a}, \mathrm{b}$, and g standards are the most common for home wireless access points and large business wireless systems [3].
WLANs have gained strong popularity in some markets, including the health-care, retail, manufacturing, and academic areas. These industries have profited from the productivity gains of using hand-held terminals and notebook computers to transmit real-time information to centralized hosts for processing [3].

A tablet PC may be defined as a type of notebook computer that has an LCD screen on which the user can write using a special-purpose pen, or by hands fingers [4]. This tablet PC without Keyboard and mouse external, make it easy to use by clients. The touch screen is known as user-friendly device since it 'deals' with the user when the user is working on the screen. Tablet PC detect the position of the point of touch. The design of touch screens is good for inputting simple, select and the choices are programmable. The areas of the tablet PC is defined as "Buttons" that the operator choose easy to touch them. The advantages of tablet PC applications are that each screen is customized to reflect only the valid options for each space of an operation [5]. Figure 1 shows customer order use touch screen.

\section{Design and Implementation of the System}

\section{System architecture}

1. Client/Server: is a computer architecture that divides functions into client (Requester) and server (Provider) sub-systems, with standard communication methods (TCP/IP wireless and wired) to easy the sharing of information between them. Work on a server, responding to each client in line [6] as shown in Figure 1.

*Corresponding author: Nibras Othman Abdul Wahid, Department of Mathematics and Computer Science, Graduate School of Natural and Applied Sciences, Ministry of Higher Education and Scientific Research, Iraq, Tel: 905340722743; E-mail: an_angel2011@yahoo.com

Received March 19, 2014; Accepted April 26, 2014; Published April 29, 2014

Citation: Wahid NOA (2014) Improve the Performance of the Work of the Restaurant Using PC Touch Screen. J Comput Sci Syst Biol 7: 103-107. doi:10.4172/jcsb.1000144

Copyright: (c) 2014 Wahid NOA. This is an open-access article distributed under the terms of the Creative Commons Attribution License, which permits unrestricted use, distribution, and reproduction in any medium, provided the original author and source are credited. 


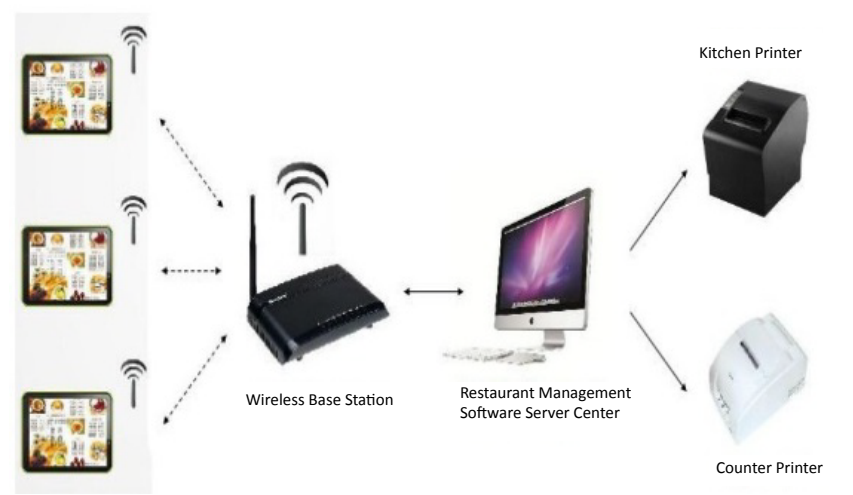

Figure 1: The system Architecture.

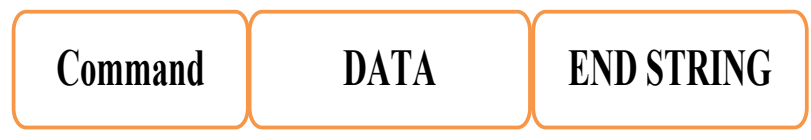

Figure 2: Package Pattern.

\begin{tabular}{|l|l|l|}
\hline $\begin{array}{l}\text { Command } \\
\text { abbreviation }\end{array}$ & Command name & Description \\
\hline Conn & Connection & For connecting with Server \\
\hline Disc & Disconnection & $\begin{array}{l}\text { For cancelling connection with } \\
\text { Server }\end{array}$ \\
\hline Order & Request Order & For food ordering \\
\hline Cancel & Cancel Oder & For menu cancelling \\
\hline Update & ADD To Order & For updating menus \\
\hline Ackn & Response from Server & $\begin{array}{l}\text { For responding to different orders } \\
\text { (Server) }\end{array}$ \\
\hline
\end{tabular}

Table 1: Command Table.

2. Access point (AP): is a device that allows wireless devices to connect to a wired network using (Wi-Fi). (Wi-Fi) enables the user to deploy a computer network without needing to run cable throughout the facility [7].

3. Customer touch screen (TS): connect it with server by (AP), by protocol (TCP/IP wireless IEEE 802.11) [7], after that can customer choose meal to touching on screen and goes order to room cooking and cashier.

4. Printer: is device to printed order or bill, printer connecting with server by cable (USB or Wireless).

5. The network operations are based on the design of protocol in the application layer for different functions in the restaurant system. The package comprises command, data and end string [8]. Figure 2 shows Package Pattern.

In the Table 1 below we include commands between Parts of the system.

\section{Tablet PC}

By the "tablet PC" the customer can choose order, he/she finds all information food. The customer can add and remove from his/her list. When customer finished to choose food from list, this order go to tow parts kitchen and cashier with more detail about this order for example Table NO., price and food items. Scheme below shows how customer use tablet to order food. Figure 3 show Process the customer's order.

\section{System design}

1. A database (DB) is one or more lists of values. A computer (DB) is one whose values are stored in a computer medium such as a hard disk or another store device. A desktop database is one that is used in one computer. A client/server DB is a DB that is stored in one computer named a server and other computers named clients connect to the server to access and use the DB [9].

2. Entity Relationship Diagram (ERD) the Restaurant includes items, Manager, Cooker, Waiter, Cashier, Customers, Menu, items or foods and Tables. Those items are real world. We will convert the real world to Entities then we find the relationship between entities. An entity type name is a singular noun. Entities in one entity type have the sane attributes [10]. Figure 4 shows the change the real world to entities.

We can be classified these entities into four groups (Entity type):-

-Person: Staff.(Entity Type)\{Manager, Chef, cashier, waiter,...\}

-Object: Customer Tables (Entity Type) \{Table 1, Table 2,.....

-Object: Food Items (Entity type) \{Pizza, Entrees, Hamburger,...

-Object: Order detail (Entity type) \{Order 1, Order 2,....\}

-Order (Relationship between entities)

3. The ER model a method to describe a database, and the relation

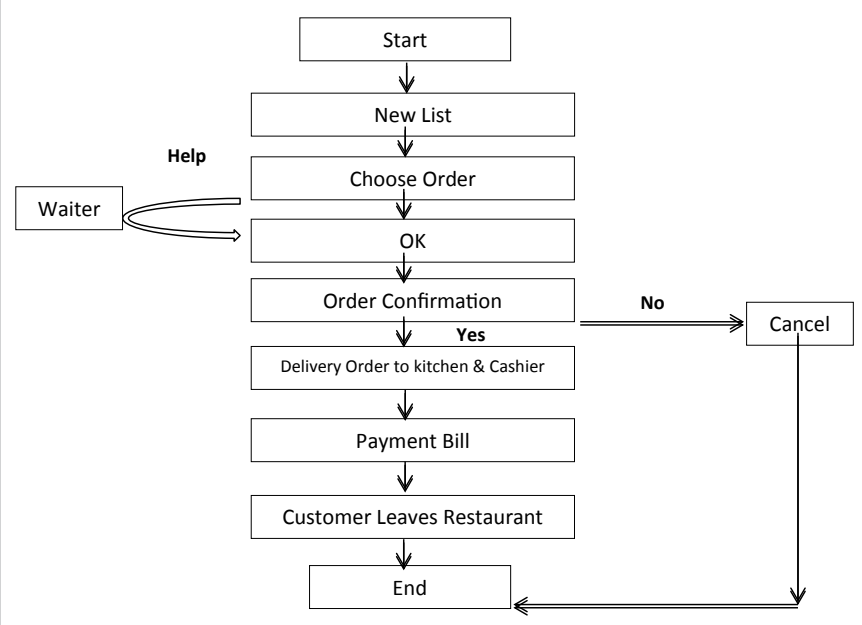

Figure 3: Scheme shows how customer use tablet PC to order food.

\section{Real World}

1-Manager. 2. Chef 3. Waiter 4- Cashier 5- Menus 6- Customer 7- Customer Tables 8- Orders. 9- Food Items 10- Sales 11-Bills

\section{$\downarrow$ Conversion To ... \\ Entities}

Figure 4: Entities 
Citation: Wahid NOA (2014) Improve the Performance of the Work of the Restaurant Using PC Touch Screen. J Comput Sci Syst Biol 7: 103-107. doi:10.4172/jcsb.1000144

between the databases. This model shows the links between entities via points or keys. Figure 5 shows the relationship between entities.

\section{The Implementation of the system}

The whole system was built using the Microsoft visual basic 6.0. Through Visual basic Environment we can build or design interfaces, through which the interfaces can users, used it, for example the user can call data, update and delete and. the database was served by Microsoft SQL server 2000. The functions of the system divided to 4 tasks: system management, cashier management, kitchen management and customer management. The system management setting and control to add, remove and edit items from food list, after the manager add or remove any items from meals, directly update in the tablet PC. In addition the manager can add and remove employees. These tasks are shown in Figures 6-8.

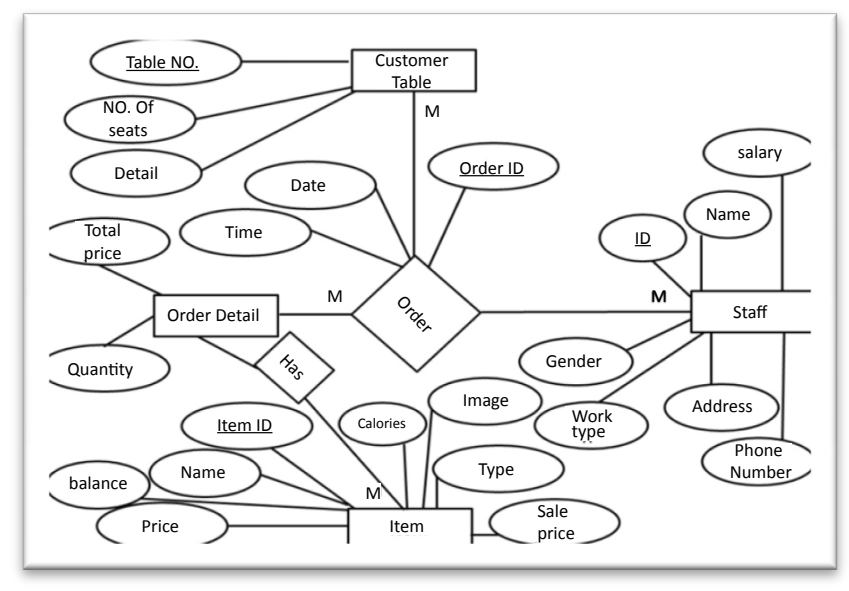

Figure 5: ER Diagram

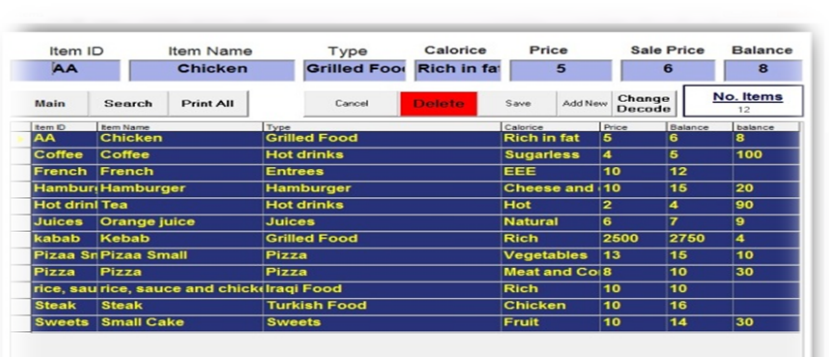

Figure 6: Add and Remove Items.

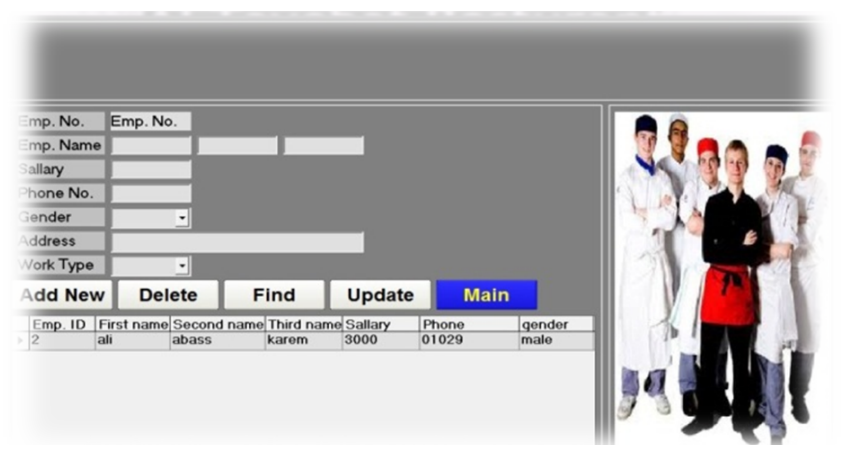

Figure 7: Add and Remove Employees.

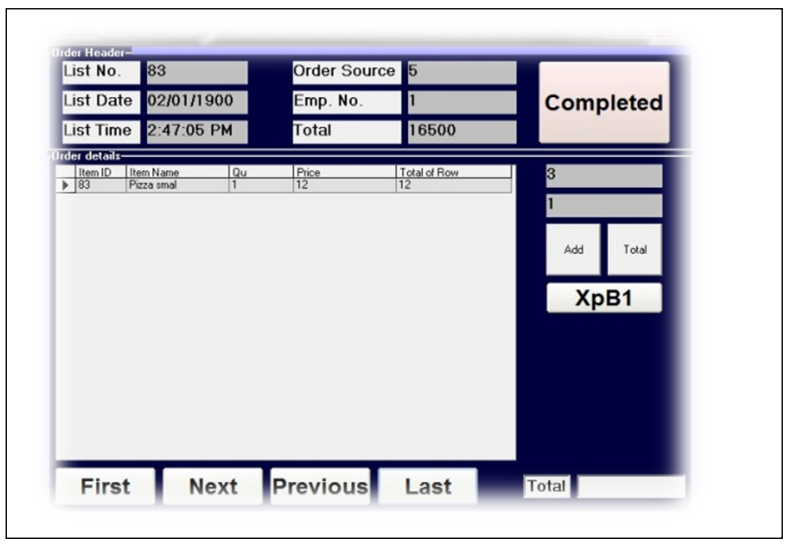

Figure 8: Kitchen Interface.

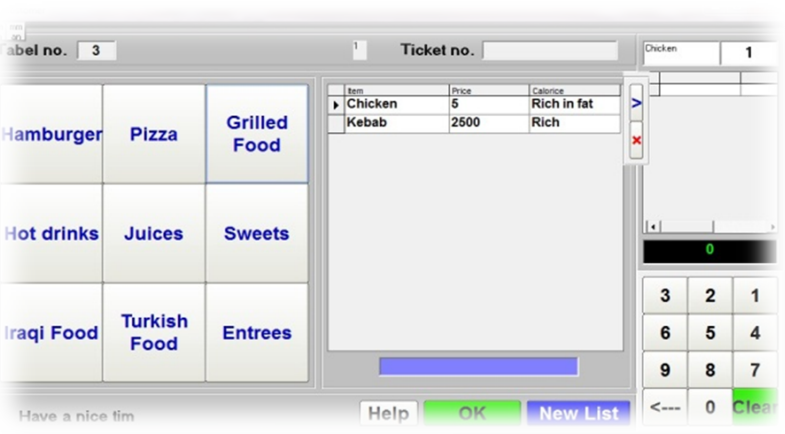

Figure 9: Customer Interface.

Add Employee

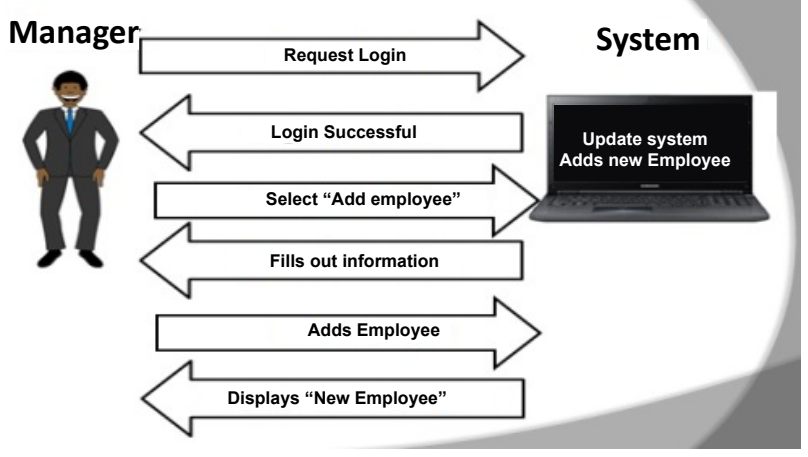

Figure 10: Add Employee.

The kitchen management after the customer chose order, and then this order send to kitchen to prepare food, this order has information about items food, quantity, calories and table NO. The cashier management receives all bills and the cashier can recognize all bills by number, each bill has id number (unique), this id is same table number. The customer management, the customer can choose his/her food by tablet PC, in case he/she cannot how use the tablet PC can call waiter by button in the customer interface. Figure 9 shows customer interface.

\section{System Sequence Diagrams}

We have three four important in our system are manager, Waiter, 
Citation: Wahid NOA (2014) Improve the Performance of the Work of the Restaurant Using PC Touch Screen. J Comput Sci Syst Biol 7: 103-107. doi:10.4172/jcsb. 1000144

Customer and PC Touch Screen. Figures 10-15 below shows how our system works.

\section{Takeaway Interface}

Our system, has more options provide to customers, like

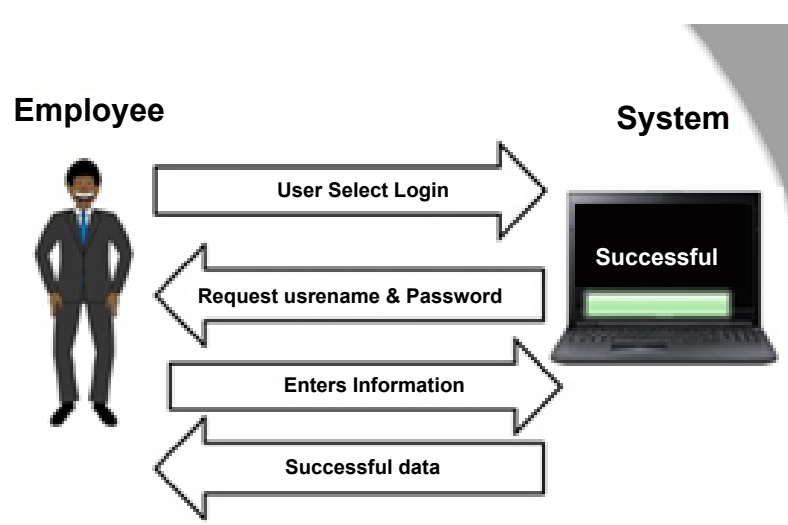

Figure 11: Employee Login

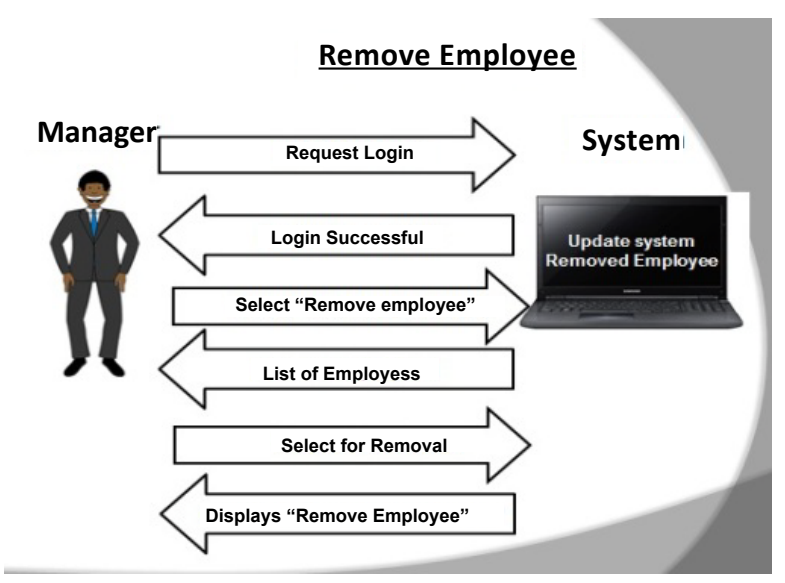

Figure 12: Remove Employee.

\section{Manage menu}

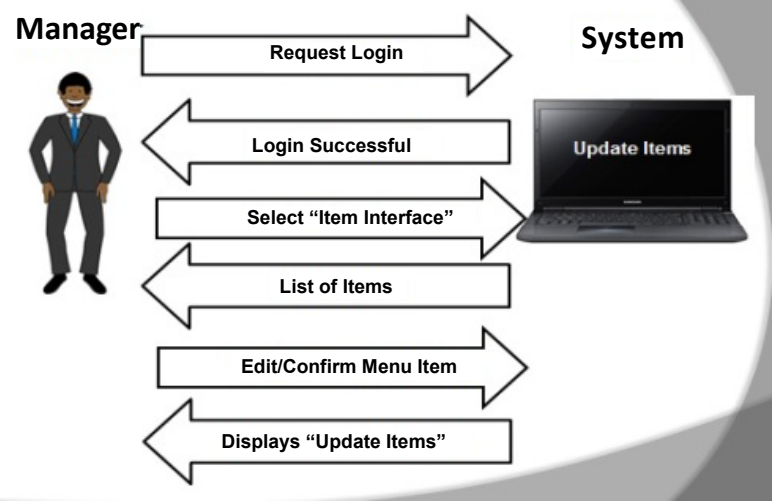

Figure 13: Manage Menu.

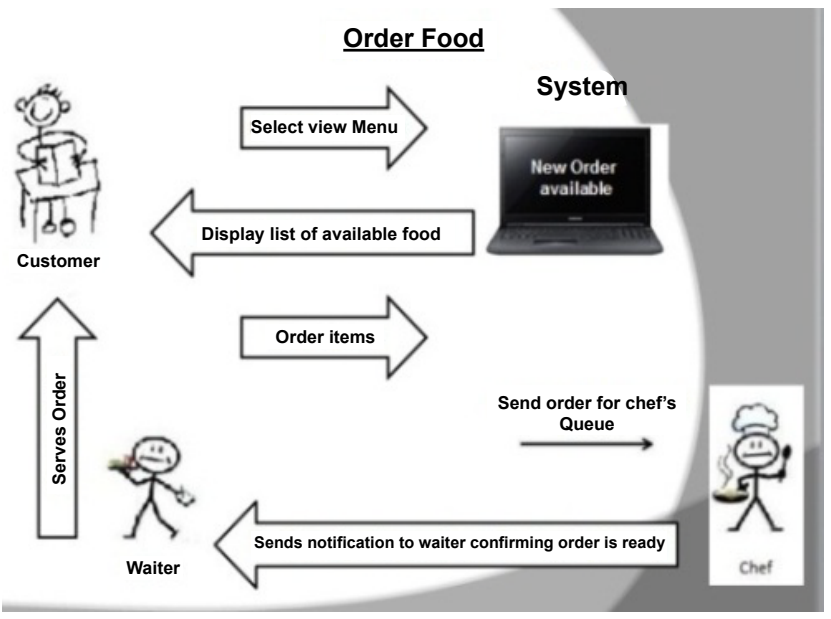

Figure 14: Customer Order.

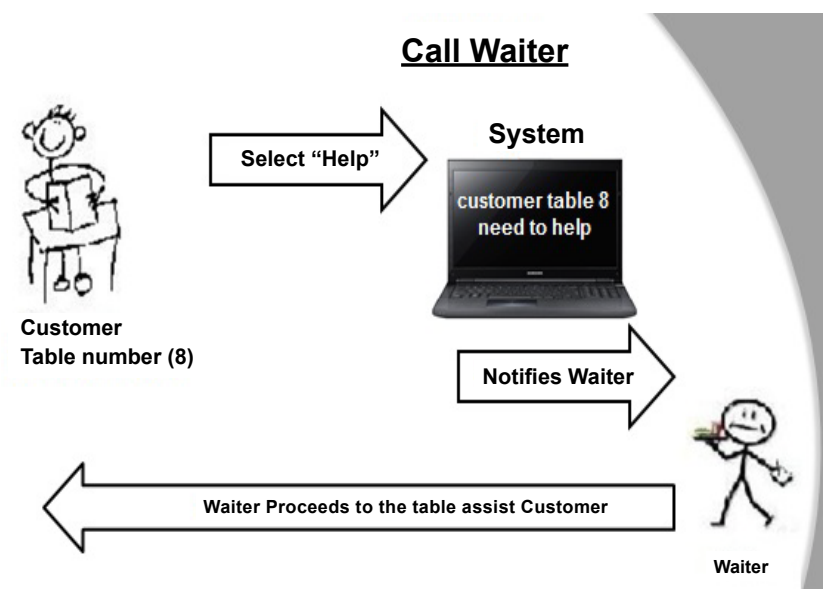

Figure 15: Call Waiter.

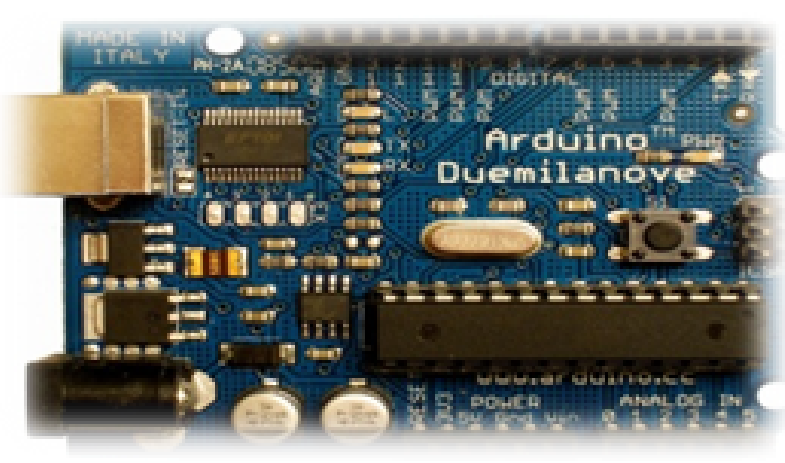

Figure 16: Arduino device.

Takeaway, by this interface the customer can chooses order, for service "Takeaway". When customer chooses order, then customer gets on ticket content on number, when the ticket number appear on screen, after that, customer receiving his/her meal. We used in this part the Arduino Hardware and LED Screen to display the customer No. on screen. Figures 16 and 17 have shown the Arduino device and LED screen. 


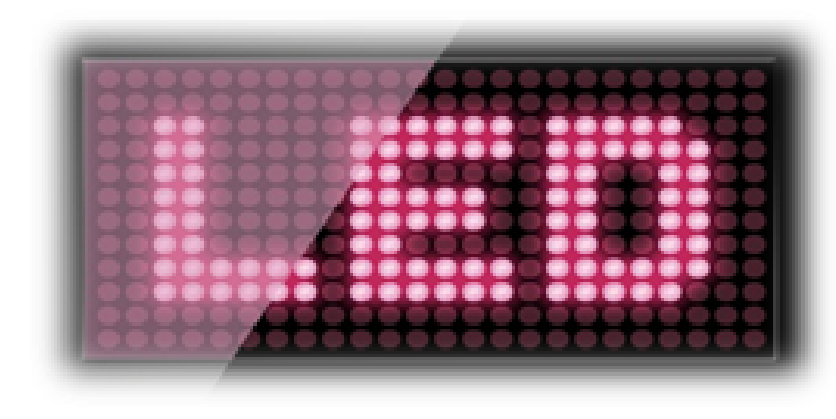

Figure 17: LED screen.

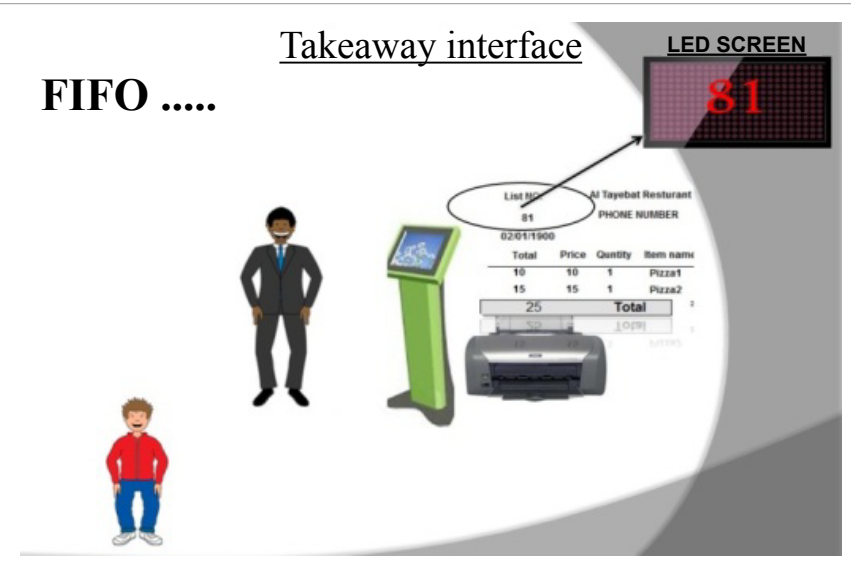

Figure 18: Takeaway.

Arduino is a single-board microcontroller, intended to make the application of interactive objects or environments more accessible. The hardware consists of an open-source hardware board designed around an 8-bit Atmel AVR microcontroller, or a 32-bit Atmel ARM. Current models feature an USB interface, 6 analog input pins, as well as 14 digital I/O pins which allow attaching various extension boards. Arduino software written by Programming Languages using $\mathrm{C}$ or $\mathrm{C}++$ [4].

A LED screen is a video display which uses light-emitting diodes. An LED panel is a small display, or a component of a larger display or screen. They are typically used outdoors in store signs and billboards, and in recent years have also become commonly used in destination signs on public transport vehicles. LED panels are sometimes used as form of lighting, for the purpose of general illumination, task lighting, or even stage lighting rather than display [5].

In Figure 18 below shows how customer cuts the ticket from the Touch Screen, After that the customer waiting the order, when the his/her order appears on the LED screen, then customer can gives the ticket to cashier and get his/her order, this option works to organizing row clients.

\section{Conclusions}

The aim of this paper has been to develop wireless ordering system in restaurant and use modern touch screen (TS) technology. We use touch screen and provide it to the customer to choose meal directly without any help from waiters. The customer orders via (TS) by customer interface, which was designed by visual basic (VB 6.0) in a user friendly way, and by utilizing the user interface tools of VB. The restaurant owner benefits from this system either, because he/she can manage the restaurant from a central computer, he/she can check the input and output of food. Restaurant owner or admin authorizes the cashier to enter to system, each waiter or cashier has username and password. This type of security has been considered in the developed system. The customer can request help in case he/she can't or they do not know how to use touch screen by (Help button) which appears on customer interface. We used wireless service in this project to connect customer tablet pc or touch screen with server database. Through (TCP/ IP wireless 802.11 standard) we can connect tablet pc and access point (AP) device, to delivery customer meal with table number (packet) to server, this is carried out using a client/server indoor application. Client initiates request and server services the request. The server has a number of security features, such as logins, Server Roles, Linked Servers and remote Servers.

By using this technique to solve the problem in traditional restaurants, the customers do not need to ask waiters what drink and food the restaurant offers. The customer finds all food and drinks in customer interface. Besides, he/she finds price of each type of food, drink and time of preparing.

As the future work the customer will be able to pay bills from tablets, which means that customer can use visa card to pay. We can make the system multi-language which means any customer can choose his language. (For example English, Arabic, etc.). Another future work for this system is applying it in five Star hotels. We can put in each room in hotel a tablet pc and the customer can request meal using this tablet, each tablet connects with access point in each floor.

\section{Acknowledgment}

I would like to thank the Minister and the employees of Ministry of Higher Education and Scientific Research who have helped enrich my knowledge and for all their support and encouragement. Finally I would like to thank my husband and my family for all their support and belief in me and for being a knack boosting morale during rough times.

\section{References}

1. Luke Atkinson, Joules Hayes, Jon-Pierre Sunderland (2013) Electronic Menu and Ordering System.

2. Sean Chang, Salman Hossin, Parimal Nasa, Michael Naumis (2007) Electronic Menu and Ordering System for Full-service Restaurants. CSC318H5 - The Design of Interactive Computational Media.

3. Mike Gunderloy, Joseph LJ, David WT, (2005) Mastering Microsoft SQL Server 2005. $1^{\text {st }}$ edition John Wiley \& Sons, 2006.

4. http://www.vegasledscreens.com/.

5. http://www.arduino.cc/.

6. Silvano Maffeis (1997) Client/Server Term Definition. International Thomson Computer Publishing, 1998.

7. Avinash Patil (2010-2011) Visvesvaraya Technological University.

8. Rob R. Weitz, Bert Wachsmuth, Danielle Mirliss (2006) The Tablet PC For Faculty: A Pilot Project. Educational Technology \& Society 9: 68-83

9. (2009) Intelligent Computation Technology and Automation. $2^{\text {nd }}$ International conference (ICICTA 2009)

10. Abraham Silberschatz, Henry FK, Sudarshan S (2006 ) Database system concepts. $5^{\text {th }}$ edition, the University of California: McGraw-Hill Higher Education. 